\title{
Concomitant Use of Statins in Tocilizumab-Treated Patients with Rheumatoid Arthritis: A Post Hoc Analysis
}

Martin Soubrier · Jinglan Pei · Frédérick Durand · Lars Gullestad ·

Ani John

Received: September 15, 2016 / Published online: November 29, 2016

(C) The Author(s) 2016. This article is published with open access at Springerlink.com

\section{ABSTRACT}

Introduction: Patients with rheumatoid arthritis (RA) have decreased survival because of increased cardiovascular risk compared with the general population, and treatment with tocilizumab (TCZ) has been shown to increase lipid levels; however, the relationship between lipids and cardiovascular risk is unknown. This post hoc analysis expanded on previously

Enhanced content To view enhanced content for this article, go to http://www.medengine.com/Redeem/A51 7F0604525E899.

Electronic supplementary material The online version of this article (doi:10.1007/s40744-016-0049-8) contains supplementary material, which is available to authorized users.

M. Soubrier ( $\square)$

Department of Rheumatology, Centre Hospitalier Universitaire de Clermont-Ferrand, Hôpital Gabriel-Montpied, Clermont-Ferrand, France e-mail: msoubrier@chu-clermontferrand.fr

J. Pei · A. John

Genentech, Inc, South San Francisco, CA, USA

F. Durand

LILLY FRANCE, Neuilly-sur-Seine, France

L. Gullestad

Department of Cardiology, Faculty of Medicine, and Oslo University Hospital, University of Oslo, Oslo, Norway reported 24-week results by characterizing statin use and subsequent changes in lipid parameters in patients with RA treated with intravenous or subcutaneous TCZ (TCZ-IV or TCZ-SC) over 2 years of treatment.

Methods: Data were collected from patients with moderate to severe active RA who received $\geq 1$ dose of the study drug in seven international, randomized, double-blind, controlled phase 3 and 4 clinical trials of TCZ-IV or TCZ-SC. Lipid levels and safety events were assessed over 2 years of treatment. Data were summarized for all pooled treatment groups of the intention-to-treat populations in the TCZ-IV and TCZ-SC studies, and results were stratified by concomitant statin use.

Results: Data from this descriptive, retrospective, pooled analysis indicated that statins can stabilize lipid levels without a clinically significant increase in adverse events. Approximately $30 \%$ of patients in the TCZ treatment arms who never received a statin demonstrated a shift in low-density-lipoprotein cholesterol (LDL-C) from $<130 \mathrm{mg} / \mathrm{dl}$ at baseline to $\geq 130 \mathrm{mg} / \mathrm{dl}$ at 2 years. However, despite the increased potential cardiovascular risk, $<15 \%$ of patients with LDL-C $\geq 100 \mathrm{mg} / \mathrm{dl}$ 
and $<35 \%$ of patients with a total cholesterol:high-density-lipoprotein cholesterol ratio $>5$ at 2 years were receiving concomitant statins.

Conclusion: Concomitant statin use attenuated TCZ-mediated lipid increases; however, a large proportion of TCZ-treated patients potentially at risk of cardiovascular disease were untreated. These findings highlight the need for better understanding of potential risk associated with TCZ-mediated lipid elevations as well as implementation of RA-specific guidelines on the recognition and management of elevated risk of cardiovascular events in patients with RA.

Funding: F. Hoffmann-La Roche, Ltd.

Keywords: Biologicals; Cardiovascular; Disease-modifying antirheumatic drugs (DMARDs); Low-density-lipoprotein cholesterol; Rheumatoid arthritis; Statins; Tocilizumab

\section{INTRODUCTION}

Rheumatoid arthritis (RA) is a disease characterized by chronic inflammation and is associated with decreased survival compared with the general population [1]. This excess mortality in patients with RA is largely due to an increased risk of cardiovascular (CV) disease, although it remains unclear whether chronic inflammation, altered lipid levels, and/or traditional risk factors are the main drivers of the increased risk of $\mathrm{CV}$ events observed in RA [2-4]. In patients with RA, lipid levels appear to have a paradoxical relationship with $\mathrm{CV}$ disease risk [5-8]. Whereas elevated lipid levels are typically associated with an increased risk of $\mathrm{CV}$ disease in the general population, lower lipid levels appear to be associated with an increased risk of CV disease in patients with RA [5]. Evidence suggests that reductions in time-averaged disease activity may decrease the risk of CV events [9]; reductions in C-reactive protein in patients with RA may lead to improvements in high-density-lipoprotein cholesterol (HDL-C) efflux and increases in apolipoprotein AI, which may counteract any potential elevated $\mathrm{CV}$ risk because of concurrent increases in low-density-lipoprotein cholesterol (LDL-C) [10]. In addition, treatment with different classes of disease-modifying antirheumatic drugs (DMARDs) has demonstrated different directional changes in levels of lipid parameters and risk of CV disease; however, associations between treatment-induced alterations in lipid profiles and CV outcomes are unknown. For example, in patients with RA, treatment with tumor necrosis factor inhibitors has been shown to significantly increase HDL-C, total cholesterol (TC), and triglyceride (TG) levels without altering LDL-C levels, while treatment with the interleukin 6 receptor $\alpha$ inhibitor tocilizumab (TCZ) generally increases levels of all four of these parameters [11]. Another study found that treatment with tumor necrosis factor inhibitors was associated with decreased risk of CV events compared with conventional synthetic DMARD (csDMARD) use, but lipid levels were not assessed [12].

TCZ, a humanized monoclonal antibody that inhibits interleukin 6 receptor $\alpha$, is approved as monotherapy or in combination with methotrexate for the treatment of patients with moderate to severe RA who have had an inadequate response to $\geq 1$ csDMARD. TCZ has proven to be safe and efficacious when used in combination with csDMARDs or as monotherapy [13-20]. Several clinical trials have investigated the effects of intravenous TCZ (TCZ-IV) or subcutaneous TCZ (TCZ-SC) therapy on lipid levels in patients with RA and 
generally found elevations in TC, LDL-C, HDL-C, and TGs by $\approx 12-16$ weeks in patients treated with TCZ, which remained stable over time (Table S1) [13, 15-30]. In addition, an integrated safety report from five randomized controlled trials and their long-term extension studies (AMBITION, RADIATE, TOWARD, OPTION, LITHE) demonstrated that lipid levels were elevated in the TCZ all-exposed population as early as 6 weeks after the first TCZ infusion and remained at these levels throughout 104 weeks of treatment [31]. Another retrospective analysis examined the associations of baseline and on-treatment lipid levels, inflammation, and disease activity with risk of major adverse CV events in TCZ-treated patients pooled from five randomized controlled trials and their extension studies. This study found that changes in measures of RA disease activity, but not necessarily changes in lipids, were associated with an increased incidence of major adverse $\mathrm{CV}$ events in TCZ-treated patients [32]. Notably, TCZ doses as high as $20 \mathrm{mg} / \mathrm{kg}$ had no impact on LDL-C levels in healthy volunteers [33]. Whether these lipid elevations actually increase or decrease the risk of CV disease in TCZ-treated patients with RA is unclear; however, patients may benefit from treatment with lipid-lowering therapy, such as statins.

The identification of potential statin-related adverse events (AEs) was initially evaluated in TCZ-treated patients who were or were not treated with concomitant statins at baseline, over 24 weeks using pooled data collected from five phase- 3 clinical trials of TCZ-IV in patients diagnosed with moderate to severe RA according to the 1987 American College of Rheumatology classification criteria [34, 35]. Overall, treatment with TCZ plus csDMARDs or as monotherapy resulted in increased mean LDL-C levels. The initial magnitude of the increase in mean LDL-C levels over the first 6 weeks was lower in TCZ-treated patients treated with concomitant statins from baseline than in the overall TCZ-treated population; the initiation of statin treatment post-baseline reduced mean LDL-C levels below baseline values in TCZ-treated patients. The original analysis included only studies of TCZ-IV. TCZ-SC is now approved; therefore, it is also important to evaluate TCZ-SC in patients with RA, especially to assess whether management of hyperlipidemia has evolved between the time of the initial pivotal trials of TCZ-IV and the more recent trial investigating TCZ-SC $[18,19]$. The objectives of this post hoc analysis were to expand on the previous 24-week results by characterizing statin use in patients with RA who were treated with TCZ and to describe subsequent changes in lipid parameters stratified by concomitant baseline statin use, using data from seven different international phase 3 and 4 clinical trials with TCZ-IV and TCZ-SC treatment arms.

\section{METHODS}

\section{Study Population}

In this post hoc analysis, data were collected from patients who received $\geq 1$ dose of study drug in seven international, multicenter, randomized, double-blind, controlled phase 3 and 4 clinical trials (OPTION, TOWARD, AMBITION, LITHE, ADACTA, SUMMACTA, RADIATE), which were designed to evaluate the safety and efficacy of TCZ-IV or TCZ-SC in patients with moderate to severe active RA $[13-17,19,20]$. Data from the seven TCZ-IV studies and the 24-week double-blind and 72-week open-label periods of SUMMACTA were pooled into four treatment groups: (1) 
csDMARD monotherapy, (2) TCZ-IV $8 \mathrm{mg} /$ kg q4w monotherapy, (3) TCZ-IV $8 \mathrm{mg}$ / $\mathrm{kg} q 4 \mathrm{w}+\mathrm{csDMARD}$ combination therapy, and (4) TCZ-SC $162 \mathrm{mg} \quad \mathrm{qw}+\mathrm{csDMARD}$ combination therapy in order to primarily examine the effects of equi-effective TCZ doses (TCZ-IV $8 \mathrm{mg} / \mathrm{kg}$ q4w and TCZ-SC $162 \mathrm{mg}$ qw). The data within each treatment group were analyzed for patients who were on a statin at baseline, initiated a statin post-baseline, or never received a statin at any point during the study.

All procedures followed were in accordance with the ethical standards of the responsible committee on human experimentation (institutional and national) and with the Helsinki Declaration of 1975, as revised in 2000 and 2008. Informed consent was obtained from all patients for being included in the studies.

\section{Clinical Assessments}

Fasting lipid levels were assessed at baseline, 3-4, 6, 12 months, and 2 years; data were summarized for all pooled treatment groups of the intention-to-treat populations in the TCZ-IV and TCZ-SC studies. Demographic characteristics and inflammatory biomarker levels-including C-reactive protein, serum amyloid A, haptoglobin, and lipoprotein(a)were assessed at baseline. Notably, patients in the TCZ-IV treatment arms were followed for up to 5 years; however, due to the small numbers of patients at these longer time points, only the lipid level assessments from the first 2 years of follow-up were included in this analysis.

Guidelines for initiation of lipid-lowering therapy vary by study site locations; however, the decision to start a statin or other lipid-lowering drug was at the discretion of the investigator and not mandated by the individual study protocols (Table S2). For this analysis, LDL-C $\geq 100 \mathrm{mg} / \mathrm{dl}$ or TC:HDL-C ratio $>5$ was used as a crude predictor of $\mathrm{CV}$ risk. To estimate whether statins were appropriately given in at-risk patients, we calculated the proportion of patients above each threshold who were on statins at each visit.

Selected AEs that were chosen post hoc as AEs that may, but not necessarily or causally, be related to statin use were assessed for up to 5 years during the individual studies and included musculoskeletal and connective tissue disorders, arthralgia, musculoskeletal pain, and myalgia. Serious CV events included all preferred terms in the Medical Dictionary for Regulatory Activities within the "cardiac disorders" and "vascular disorders" system organ classes.

\section{Statistical Analysis}

Descriptive statistics were used to summarize laboratory data, statin use, and AEs at each visit, and the results were stratified by concomitant statin use. All laboratory data were converted to the International System of Units and summarized with actual values and with the change from baseline over visits. Abnormal laboratory values were classified according to the National Cancer Institute's Common Terminology Criteria for Adverse Events grading system (version 3) [36]. Shift tables summarized the proportion of patients at each visit with categorical shifts in lipid parameters from normal values at baseline (LDL-C $<130 \mathrm{mg} / \mathrm{dl} ;$ TC $<240 \mathrm{mg} / \mathrm{dl} ;$ HDL-C $<60 \mathrm{mg} / \mathrm{dl} ; \quad \mathrm{TG}<150 \mathrm{mg} / \mathrm{dl}$ ) to the highest post-baseline values (LDL-C $\geq 130 \mathrm{mg} / \mathrm{dl}$; TC $\geq 240 \mathrm{mg} / \mathrm{dl}$; HDL-C $\geq 60 \mathrm{mg} / \mathrm{dl} ; \mathrm{TG} \geq 150 \mathrm{mg} /$ dl).

All selected AEs and serious CV events were presented as event rates per 100 patient-years 
with 95\% confidence intervals. No statistical imputation was performed for missing visits or incomplete data.

\section{RESULTS}

\section{Baseline Characteristics}

In this post hoc analysis, statin use and lipid levels were analyzed over time in 4655 patients with RA pooled from the seven phase 3 and 4 TCZ-IV and TCZ-SC clinical trials [CsDMARD monotherapy $(n=1361)$, TCZ-IV $8 \mathrm{mg} / \mathrm{kg}$ q4w monotherapy $\quad(n=450), \quad$ TCZ-IV $8 \mathrm{mg} / \mathrm{kg}$ $\mathrm{q} 4 \mathrm{w}+$ csDMARD combination therapy $(n=2213)$, TCZ-SC $162 \mathrm{mg}$ qw $(n=631)]$. Baseline demographic and clinical characteristics for all patients pooled from the seven phase 3 and 4 TCZ-IV and TCZ-SC clinical trials stratified by baseline statin use are presented in Table 1. Patients on statins at baseline in the TCZ-IV and TCZ-SC studies were generally older and weighed more compared with patients not treated with statins at baseline (Table 1). A higher proportion of patients on statins at baseline were male, had diabetes, had a family history of coronary heart disease, and had a history of hypertension and cardiac disorders compared with patients who were not treated with statins at baseline. These trends were similar in the TCZ-IV and TCZ-SC treatment groups (Table 1).

\section{Description of Statin Use Over Time}

In this post hoc analysis of 4655 patients, including 2616 patients (56.2\%) with elevated LDL-C levels $\geq 100 \mathrm{mg} / \mathrm{dl}$ at baseline, 443 patients $(9.5 \%)$ were on concomitant statins at baseline, 264 patients (5.9\%) initiated statins post-baseline, and 3948 patients (84.8\%) never initiated a statin during the study period. Baseline statin use was comparable across all treatment groups (range 8.0-11.4\%) (Table S3). The most common lipid-lowering agents used at all time points were the lipophilic agents simvastatin and atorvastatin, with $3.2-4.6 \%$ of patients in each arm receiving simvastatin and $2.7-3.2 \%$ of patients receiving atorvastatin. Over time, statin use generally increased in the TCZ treatment groups and was three- to fourfold higher at 2 years (range 13.8-17.4\%) compared with patients receiving csDMARD monotherapy (4.5\%).

\section{Analysis of Lipid Parameters}

\section{Evolution of LDL-C Over Time by Statin Use}

Statin use at baseline appeared to attenuate the increase in LDL-C over time. Of patients who were on a statin at baseline, mean (SD) LDL-C levels ranged from 98.3 (34.6) to 101.9 (29.0) $\mathrm{mg} / \mathrm{dl}$, with no clear differences across treatment groups based on overlapping confidence intervals (Fig. 1a). Of patients who initiated a statin post-baseline, mean (SD) LDL-C levels ranged from 129.1 (32.4) to 144.6 (42.6) $\mathrm{mg} / \mathrm{dl}$, with the lowest values observed in the TCZ-IV monotherapy treatment group (Fig. 1b). Patients who were untreated with statins at any time point during the study had baseline LDL-C levels in between those of patients who were on statins at baseline and those who initiated statins post-baseline, with mean (SD) levels ranging from 109.1 (32.9) to 114.9 (33.5) mg/dl (Fig. 1c). Although LDL-C levels at baseline were highest in patients who initiated statins post-baseline, the magnitude of the LDL-C increase over the first 3-4 months of TCZ treatment was similar across treatment groups. Evaluation of mean TC, HDL-C, and TG levels over time for patients on statins at baseline, those initiating statins post-baseline, 


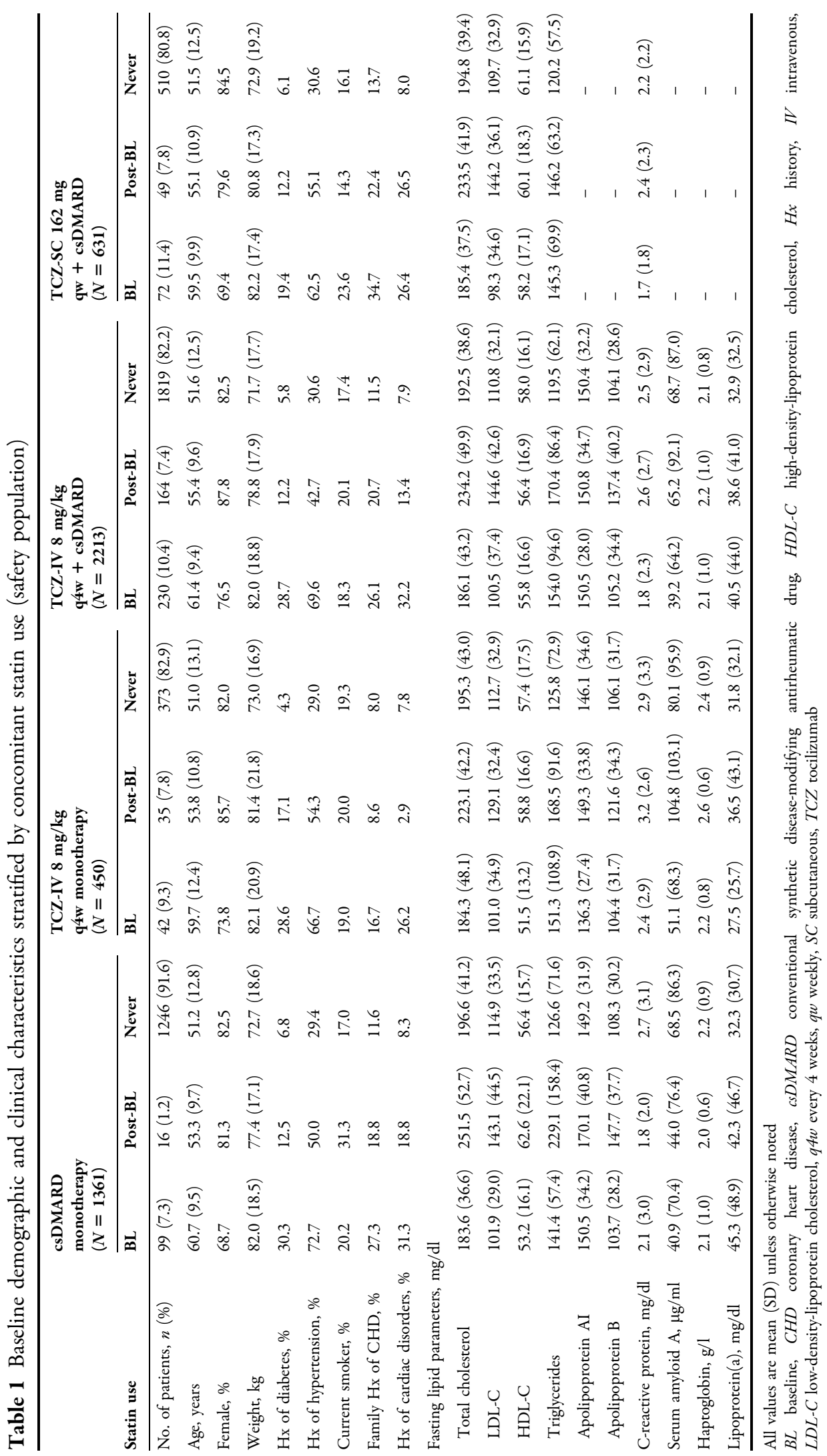





Fig. 1 Mean LDL-C levels over time in patients who were on statins at baseline (a), patients who initiated statins post-baseline (b), and patients who were untreated with statins at any time (c). csDMARD conventional synthetic

and those untreated with statins at any time is presented in the Supplemental Material (Figs. S1-S3). The observed trends in TC, HDL-C, and TG changes were generally similar to those seen for LDL-C. Importantly, patients who were on concomitant statins at baseline or who never received statins during the study period exhibited persistent elevations in lipids after 3-4 months, whereas those patients who initiated statins post-baseline demonstrated gradual decreases in lipid levels over time, signaling a possible trend toward normalization of previously elevated lipids.

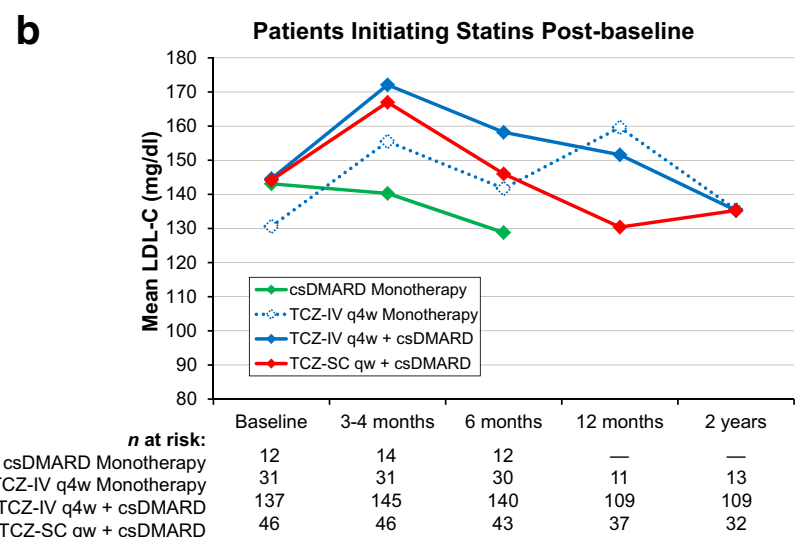

disease-modifying antirheumatic drug, $I V$ intravenous, $L D L-C$ low-density-lipoprotein cholesterol, $q w$ every week, $q 4 w$ every 4 weeks, $S C$ subcutaneous, $T C Z$ tocilizumab

\section{Lipid Shifts from Baseline to Last Observation by Statin Use}

Shifts in lipid parameters from normal values at baseline to the highest post-baseline values over time are presented for patients stratified by statin use (Table 2). Of patients who were on statins at baseline, 31.8\% (7/22), 24.8\% (34/ $137)$, and $29.8 \%(14 / 47)$ of patients receiving TCZ-IV as monotherapy, TCZ-IV plus csDMARDs, or TCZ-SC, respectively, demonstrated an increase in LDL-C from $<130 \mathrm{mg} / \mathrm{dl}$ at baseline to $\geq 130 \mathrm{mg} / \mathrm{dl}$ at 3-4 months. 


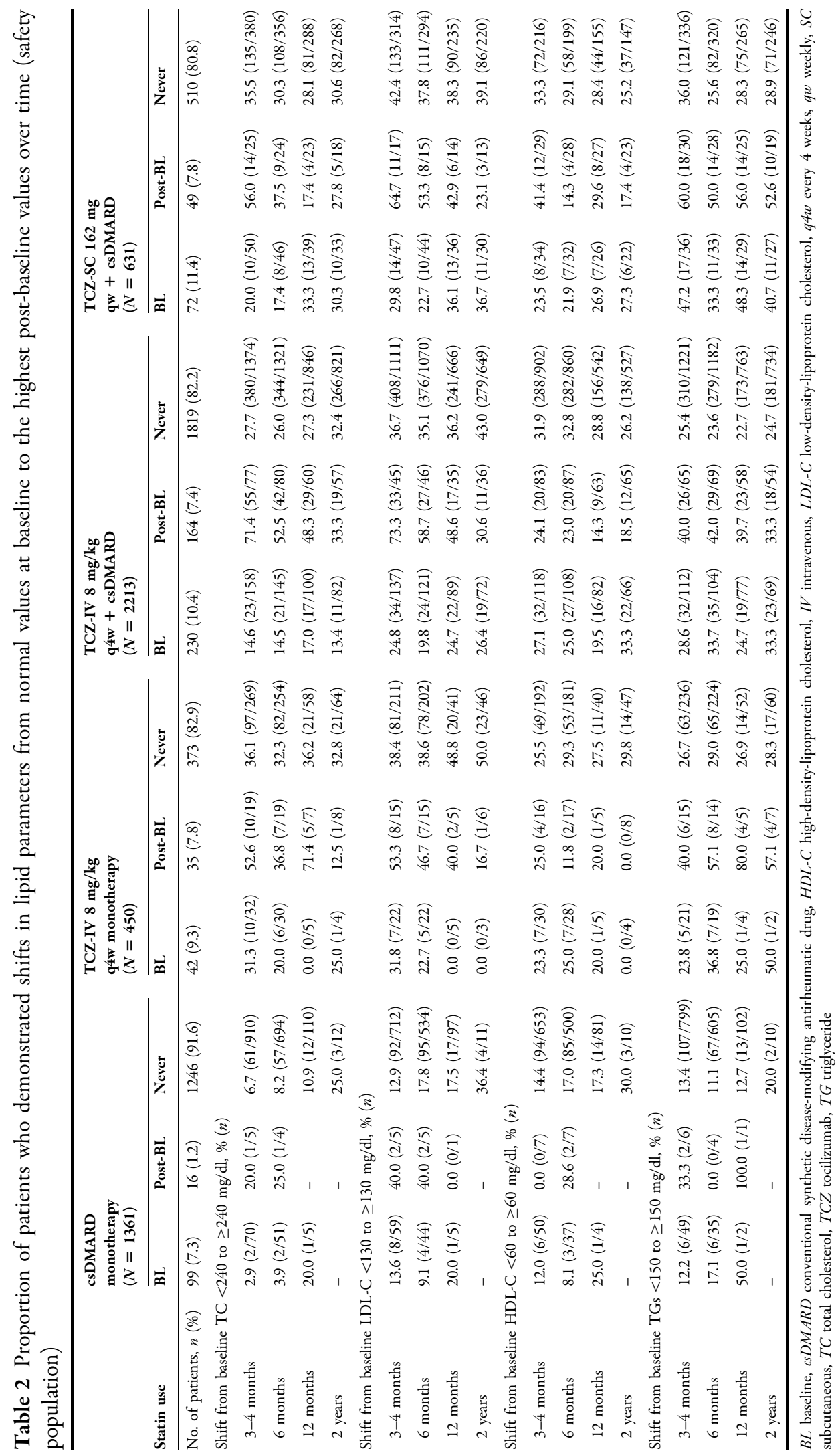


Similar trends were observed for TCZ-treated patients who were untreated with statins at any time; however, as expected, a higher proportion of patients overall exhibited a shift from LDL-C $<130 \mathrm{mg} / \mathrm{dl}$ at baseline to LDL-C $\geq 130 \mathrm{mg} / \mathrm{dl}$ (Table 2). Of those never treated with statins, $38.4 \%$ (81/211), 36.7\% (408/1111), and $42.4 \%$ $(133 / 314)$ of patients receiving TCZ-IV as monotherapy, TCZ-IV plus csDMARDs, or TCZ-SC, respectively, demonstrated an increase in LDL-C from $<130 \mathrm{mg} / \mathrm{dl}$ at baseline to $\geq 130 \mathrm{mg} / \mathrm{dl}$ at $3-4$ months. These trends were consistent through 2 years of observation; however, the smaller numbers of patients followed over time preclude making any meaningful conclusions. Notably, a lower proportion of patients who were randomized to csDMARD monotherapy and were on statins at baseline or who were never treated with statins demonstrated a shift from LDL-C $<130 \mathrm{mg} / \mathrm{dl}$ at baseline to LDL-C $\geq 130 \mathrm{mg} / \mathrm{dl}$ at 3-4 months compared with any TCZ therapy $[13.6 \%(8 / 59)$ and $12.9 \%$ (92/712), respectively].

Similar trends were generally observed for categorical shifts in TC, HDL-C, and TGs by treatment group and statin use (Table 2). Compared with patients who were never treated with statins, a smaller proportion of TCZ-treated patients (any formulation) who were on statins at baseline demonstrated shifts in TC and HDL-C from normal values $(<240$ and $<60 \mathrm{mg} / \mathrm{dl}$, respectively) at baseline to elevated levels ( $\geq 240$ and $\geq 60 \mathrm{mg} / \mathrm{dl}$, respectively) through 6 months. This observation continued through 2 years for all treatment groups, except for the shift in TC in patients receiving TCZ-SC at 12 months and shifts in HDL-C in patients receiving TCZ-IV or TCZ-SC plus csDMARDs (observed through 12 months). Compared with patients who were never treated with statins, a larger proportion of patients receiving TCZ (IV or SC) plus csDMARDs demonstrated shifts in
TGs from $<150 \mathrm{mg} / \mathrm{dl}$ at baseline to $\geq 150 \mathrm{mg} / \mathrm{dl}$ through 2 years of follow-up. Statin use did not appear to have an appreciable effect on shifts from baseline in TC, HDL-C, or TGs for patients randomized to csDMARD monotherapy.

\section{Proportion of Patients Treated with Statins by Elevated LDL-C Levels and TC:HDL-C Ratio} For this analysis, LDL-C $\geq 100 \mathrm{mg} / \mathrm{dl}$ was used to define patients who should be treated with lipid-lowering agents, regardless of whether they were prescribed one or not. The proportions of patients with LDL-C $\geq 100 \mathrm{mg}$ / $\mathrm{dl}$ at baseline who were treated with statins at baseline, 3-4, 6, 12 months, and 2 years are presented in Table 3 . Overall, $\approx 60 \%$ of patients across all studies had LDL-C $\geq 100 \mathrm{mg} / \mathrm{dl}$ at baseline: only $6.3-7.1 \%$ of patients in the TCZ-IV arms and $5.4 \%$ of patients in the csDMARD treatment arms were treated with statins. At 2 years, $\approx 78 \%$ of patients in the TCZ-IV arms had LDL-C $\geq 100 \mathrm{mg} / \mathrm{dl}$; of these patients, only 11.0 and $13.3 \%$ of patients receiving TCZ-IV as monotherapy and combination therapy, respectively, were treated with statins. A higher proportion of TCZ-SC-treated patients with LDL $\geq 100 \mathrm{mg} / \mathrm{dl}$ at baseline were treated with statins at baseline $(8.6 \%)$ than those in the TCZ-IV studies; however, overall, a high proportion of patients remained untreated. The proportion of patients receiving TCZ-SC with LDL-C $\geq 100 \mathrm{mg} / \mathrm{dl}$ who were also treated with statins generally increased over time; however, only $13.6 \%$ of patients were treated with statins despite having LDL-C $\geq 100 \mathrm{mg} / \mathrm{dl}$ at 2 years.

Similarly, the proportion of patients with TC:HDL-C $>5$ (above-average risk) who were treated with statins at any time point was low (Table 3). Approximately $5-10 \%$ of patients across all treatment groups had TC:HDL-C $>5$ at baseline; of these patients, 9.3 and $9.7 \%$ of 


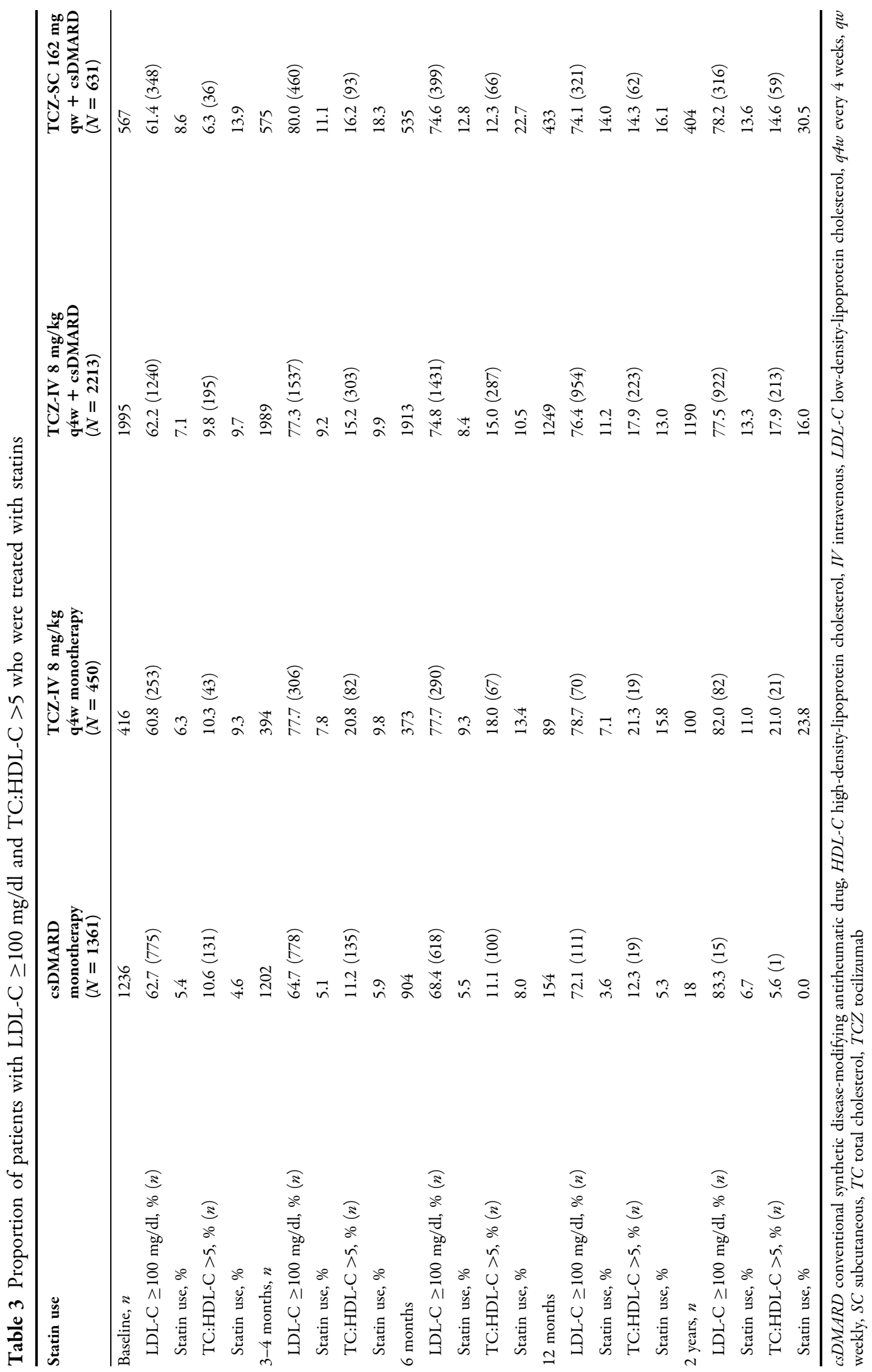


patients who were receiving TCZ-IV as monotherapy or combination therapy, respectively, were treated with statins. The proportion of TCZ-SC-treated patients treated with statins was only slightly higher (13.9\%). The proportion of patients with TC:HDL-C $>5$ generally increased over time across the studies, accounting for $\approx 15-20 \%$ of TCZ-treated patients at 2 years. Compared with patients who had LDL-C $\geq 100 \mathrm{mg} / \mathrm{dl}$, a higher proportion of patients with TC:HDL-C $>5$ were treated with statins at 2 years. Patients randomized to TCZ-SC were the most likely to be treated with statins (30.5\%), followed by TCZ-IV monotherapy (23.8\%) and TCZ-IV plus csDMARDs (16.0\%). Higher statin use in patients treated with TCZ-SC was an expected finding, because it was already known from the TCZ-IV studies that blood lipid concentrations during TCZ treatment should be monitored and treated, if needed.

\section{Safety}

The rates of selected AEs and serious CV events by statin use are summarized in Table 4 . Of patients randomized to TCZ-IV $8 \mathrm{mg} / \mathrm{kg}$ $\mathrm{q} 4 \mathrm{w}+$ csDMARDs, those who were on concomitant statins at baseline had a higher rate of $\geq 1$ musculoskeletal and connective tissue disorder, arthralgia, musculoskeletal pain, and myalgia per 100 person-years compared with patients in the same treatment arm who never received a statin during the study. For the TCZ-IV $8 \mathrm{mg} / \mathrm{kg} \quad \mathrm{q} 4 \mathrm{w}$ monotherapy and TCZ-SC $162 \mathrm{mg}$ qw treatment groups, similar rates of AEs commonly associated with statin use were observed across any of the statin groups. The low numbers of patients in the non-TCZ arms who were treated with concomitant statins precluded any meaningful comparisons to determine any effect of TCZ treatment on these safety events. The overall rates of serious cardiac disorders and vascular disorders were low and similar between treatment groups or by statin use.

\section{DISCUSSION}

This was a large, descriptive, post hoc analysis of 4655 patients enrolled in phase 3 and 4 TCZ trials. Overall, $\approx 60 \%$ of patients had baseline LDL-C levels $\geq 100 \mathrm{mg} / \mathrm{dl}$, and $9.5 \%$ of patients were on a concomitant statin at baseline. Treatment with TCZ plus csDMARDs or as monotherapy resulted in increased mean LDL-C levels in patients with moderate to severe RA, regardless of the route of administration. The initial increases in mean LDL-C levels over 3-4 months were comparable across all treatment groups; however, patients who initiated statins post-baseline experienced a subsequent decrease in LDL-C levels over time, whereas patients who were on statins at baseline or who were never treated with statins demonstrated a persistent elevation in LDL-C after 3-4 months of treatment. Similar trends were observed with TC, HDL-C, and TGs. However, despite these results, we also found that only a small proportion of patients who may be at a higher risk of $\mathrm{CV}$ disease (as estimated by LDL-C $\geq 100 \mathrm{mg} / \mathrm{dl}$ or TC:HDL-C $>5$ ) were treated with statins at any time point.

Because this was a descriptive post hoc analysis using pooled data, this study is not without some limitations. Notably, because a variety of different lipid-lowering agents were used, and at varying doses, it is difficult to ascertain the true effect of statins for the treatment groups that received concomitant statins at baseline or initiated statin treatment during the study. Similarly, it would be difficult 


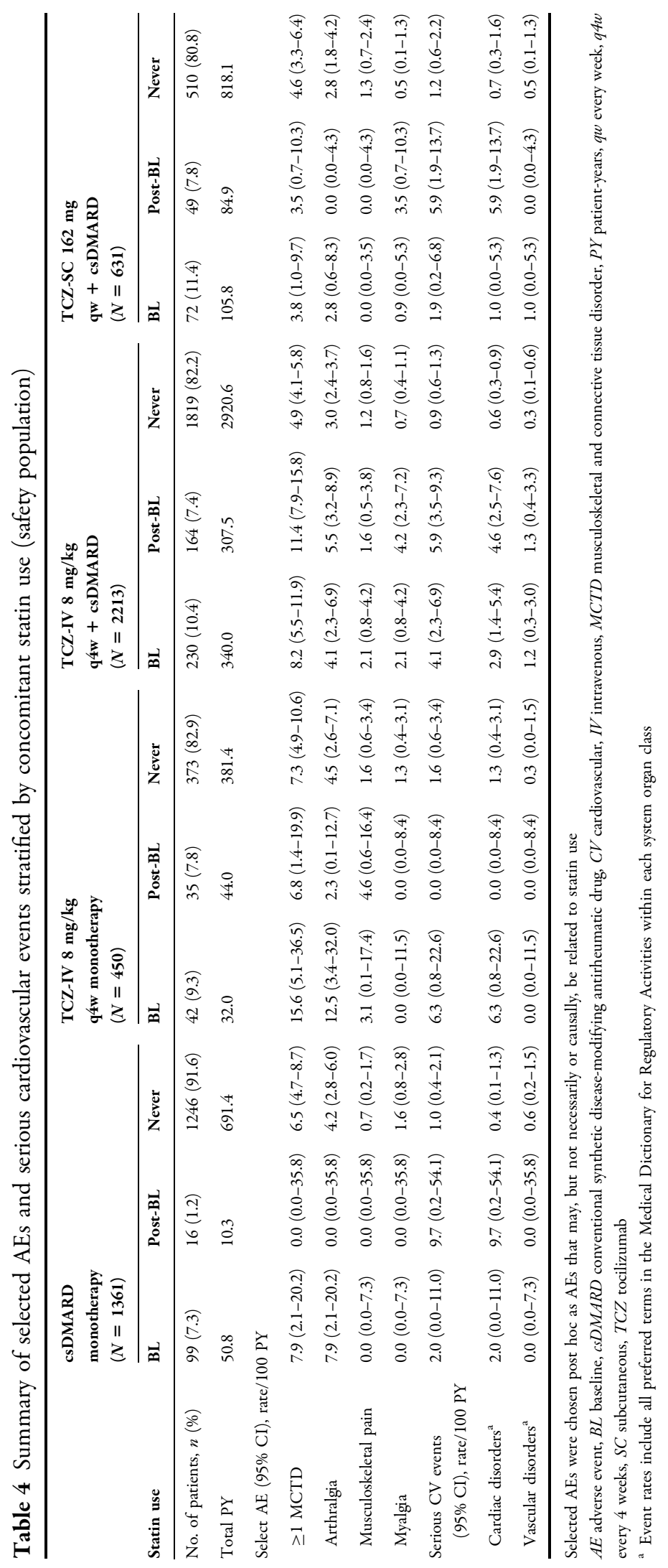


to discern whether hydrophilic statins (e.g., pravastatin and rosuvastatin) exhibit a different effect on TCZ-mediated increases in lipids compared with lipophilic statins (e.g., atorvastatin, lovastatin, simvastatin). There was also a relatively small number of patients with long-term lipid level assessments and related outcomes (up to 5 years); therefore, the lack of extended follow-up for larger numbers of patients may preclude making any meaningful conclusions about long-term trends in lipids and associations with concomitant statin use. However, a randomized, open-label, parallel-group, multicenter study designed to evaluate the rate of $\mathrm{CV}$ events over 5 years of follow-up with TCZ in comparison to etanercept in patients with RA was completed earlier this year (ClinicalTrials.gov identifier NCT01331837). Finally, the AEs assessed in this study were selected on a post hoc basis to describe events that may be related to statin use; however, there is no evidence to confirm that statin use had a causal relationship with any of these events.

LDL-C levels should be monitored and managed in patients with RA. Screening and monitoring risk factors for $\mathrm{CV}$ disease are important for preventative and management strategies in high-risk populations. The most widely used assessments of $\mathrm{CV}$ risk are the Framingham Risk Score, the Systematic Coronary Risk Evaluation, the Reynolds Risk Score, and the QRisk2 score; however, these algorithms were developed for the general population and do not account for any additional risk due to RA-specific risk factors. Unfortunately, many current risk calculators may underestimate $\mathrm{CV}$ disease risk in patients with RA, indicating a need for an RA-specific calculator to estimate risk of $\mathrm{CV}$ disease. A prospective study from an early RA inception cohort found that the Framingham Risk Score, the Systematic Coronary Risk Evaluation, and the Reynolds Risk Score primarily underestimated $\mathrm{CV}$ risk at low and moderate observed risk levels while mostly overestimating $\mathrm{CV}$ risk at higher observed risk levels [37]. The QRisk2 score generally overestimated CV risk at all levels of observed CV risk. These results highlight the need for development of an RA-specific risk model to improve prediction of $\mathrm{CV}$ risk in patients with RA and management of traditional CV risk factors [37, 38]. A recent systematic literature review to identify and appraise all $\mathrm{CV}$ disease prevention guideline recommendations and quality indicators identified a total of ten guidelines that provided recommendations for $\mathrm{CV}$ disease prevention in patients with RA published between 2008 and 2013 [39]. Of these, only the European League Against Rheumatism (EULAR) guidelines suggested a formal adjustment of the $\mathrm{CV}$ risk score to account for the presence of RA and recommended control of RA disease activity as a potential means of reducing $\mathrm{CV}$ disease risk in RA [40]. The recommendations acknowledge that RA should be regarded as a condition associated with higher $\mathrm{CV}$ disease risk. One of the recommendations notes that risk-score models should be adapted for patients with RA by introducing a $1.5 \times$ multiplication factor, and this multiplication factor should be used when patients with RA meet two of the following three criteria: disease duration $>10$ years, rheumatoid factor or anticyclic citrullinated peptide antibody positivity, and/or presence of certain extra-articular manifestations. Although there are many guidelines and quality indicators for CV risk assessment similar to the EULAR recommendations, clear gaps exist in clinical care, such as how antirheumatic treatments modify $\mathrm{CV}$ risk, which should be addressed by development of RA-specific 
guidelines [39]. A newer, expanded risk score for $\mathrm{CV}$ outcomes in patients with RA (ERS-RA) was developed to improve upon existing prediction models by incorporating RA-specific factors (e.g., RA disease activity, disability, daily prednisone use, and disease duration) in addition to traditional risk factors, allowing for more targeted management of $\mathrm{CV}$ disease in patients with RA [41].

\section{CONCLUSION}

In conclusion, data from this pooled analysis indicate that statins can stabilize lipid levels without a clinically significant increase in AEs of interest in patients treated with TCZ-IV or TCZ-SC. Approximately 30\% of patients in the TCZ treatment arms who never initiated a statin demonstrated a shift in LDL-C from $<130 \mathrm{mg} / \mathrm{dl}$ at baseline to $\geq 130 \mathrm{mg} / \mathrm{dl}$ at 2 years; however, only a small proportion of patients with LDL-C $\geq 100 \mathrm{mg} / \mathrm{dl}$ or TC:HDL-C $>5$ were treated with statins at any point during the study. TCZ is known to reduce chronic inflammation, and these results suggest that concomitant treatment with statins may attenuate lipid increases with TCZ treatment. Taken together, these findings highlight the need for better understanding of potential risk associated with TCZ-mediated lipid elevations as well as development and implementation of RA-specific guidelines on the recognition and management of elevated risk of $\mathrm{CV}$ events in patients with RA.

\section{ACKNOWLEDGEMENTS}

Funds to support this analysis and article processing charges for this manuscript were provided by F. Hoffmann-La Roche, Ltd. All named authors meet the ICMJE criteria for authorship for this manuscript, take responsibility for the integrity of the work as a whole, and have given final approval to the version to be published. All authors had full access to all of the data in this study and take complete responsibility for the integrity of the data and accuracy of the data analysis. Support for third-party writing assistance for this manuscript, furnished by Eric Deutsch, PhD, of Health Interactions, was provided by $\mathrm{F}$. Hoffmann-La Roche, Ltd.

Disclosures. Martin Soubrier has no interests to declare. Ani John is an employee of Genentech, Inc. Jinglan Pei is an employee of Genentech, Inc. Frédérick Durand was an employee of Chugai Pharma France at the time of writing. Lars Gullestad has received honoraria for advisory board meetings from Roche and ResMed.

Compliance with Ethics Guidelines. All procedures followed were in accordance with the ethical standards of the responsible committee on human experimentation (institutional and national) and with the Helsinki Declaration of 1975 , as revised in 2000 and 2008. Informed consent was obtained from all patients for being included in the studies.

Data Availability. All data generated or analyzed during this study are included in this published article (in the main document and as supplementary information). Data from the individual clinical trials have all been previously published.

Open Access. This article is distributed under the terms of the Creative Commons Attribution-NonCommercial 4.0 International License (http://creativecommons.org/licenses/ by-nc/4.0/), which permits any 
noncommercial use, distribution, and reproduction in any medium, provided you give appropriate credit to the original author(s) and the source, provide a link to the Creative Commons license, and indicate if changes were made.

\section{REFERENCES}

1. Avina-Zubieta JA, Thomas J, Sadatsafavi M, Lehman AJ, Lacaille D. Risk of incident cardiovascular events in patients with rheumatoid arthritis: a meta-analysis of observational studies. Ann Rheum Dis. 2012;71:1524-9.

2. Gabriel SE. Why do people with rheumatoid arthritis still die prematurely? Ann Rheum Dis. 2008;67(Suppl 3):iii30-4.

3. Maradit-Kremers H, Crowson CS, Nicola PJ, et al. Increased unrecognized coronary heart disease and sudden deaths in rheumatoid arthritis: a population-based cohort study. Arthritis Rheum. 2005;52:402-11.

4. Solomon DH, Kremer J, Curtis JR, et al. Explaining the cardiovascular risk associated with rheumatoid arthritis: traditional risk factors versus markers of rheumatoid arthritis severity. Ann Rheum Dis. 2010;69:1920-5.

5. Myasoedova E, Crowson CS, Kremers HM, et al. Lipid paradox in rheumatoid arthritis: the impact of serum lipid measures and systemic inflammation on the risk of cardiovascular disease. Ann Rheum Dis. 2011;70:482-7.

6. Zhang J, Chen L, Delzell E, et al. The association between inflammatory markers, serum lipids and the risk of cardiovascular events in patients with rheumatoid arthritis. Ann Rheum Dis. 2014;73:1301-8.

7. Choy E, Ganeshalingam K, Semb AG, Szekanecz Z, Nurmohamed M. Cardiovascular risk in rheumatoid arthritis: recent advances in the understanding of the pivotal role of inflammation, risk predictors and the impact of treatment. Rheumatology (Oxford). 2014;53:2143-54.

8. Gonzalez-Gay MA, Gonzalez-Juanatey C. Inflammation and lipid profile in rheumatoid arthritis: bridging an apparent paradox. Ann Rheum Dis. 2014;73:1281-3.

9. Solomon DH, Reed GW, Kremer JM, et al. Disease activity in rheumatoid arthritis and the risk of cardiovascular events. Arthritis Rheumatol. 2015;67:1449-55.

10. Liao KP, Playford MP, Frits M, et al. The association between reduction in inflammation and changes in lipoprotein levels and HDL cholesterol efflux capacity in rheumatoid arthritis. J Am Heart Assoc. 2015;4:e001588.

11. Lim DT, Cannella AC, Michaud KD, Mikuls TR. Cardiovascular risk and the use of biologic agents in rheumatoid arthritis. Curr Rheumatol Rep. 2014;16:459.

12. Greenberg JD, Kremer JM, Curtis JR, et al. Tumour necrosis factor antagonist use and associated risk reduction of cardiovascular events among patients with rheumatoid arthritis. Ann Rheum Dis. 2011;70:576-82.

13. Smolen JS, Beaulieu A, Rubbert-Roth A, et al. Effect of interleukin-6 receptor inhibition with tocilizumab in patients with rheumatoid arthritis (OPTION study): a double-blind, placebo-controlled, randomised trial. Lancet. 2008;371:987-97.

14. Emery P, Keystone E, Tony HP, et al. IL-6 receptor inhibition with tocilizumab improves treatment outcomes in patients with rheumatoid arthritis refractory to anti-tumour necrosis factor biologicals: results from a 24-week multicentre randomised placebo-controlled trial. Ann Rheum Dis. 2008;67:1516-23.

15. Genovese MC, McKay JD, Nasonov EL, et al. Interleukin-6 receptor inhibition with tocilizumab reduces disease activity in rheumatoid arthritis with inadequate response to disease-modifying antirheumatic drugs: the tocilizumab in combination with traditional disease-modifying antirheumatic drug therapy study. Arthritis Rheum. 2008;58:2968-80.

16. Jones G, Sebba A, Gu J, et al. Comparison of tocilizumab monotherapy versus methotrexate monotherapy in patients with moderate to severe rheumatoid arthritis: the AMBITION study. Ann Rheum Dis. 2010;69:88-96.

17. Kremer JM, Blanco R, Brzosko M, et al. Tocilizumab inhibits structural joint damage in rheumatoid arthritis patients with inadequate responses to methotrexate: results from the double-blind treatment phase of a randomized placebo-controlled trial of tocilizumab safety and prevention of structural joint damage at one year. Arthritis Rheum. 2011;63:609-21.

18. Kivitz A, Olech E, Borofsky M, et al. Subcutaneous tocilizumab versus placebo in combination with disease-modifying antirheumatic drugs in patients 
with rheumatoid arthritis. Arthritis Care Res (Hoboken). 2014;66:1653-61.

19. Burmester GR, Rubbert-Roth A, Cantagrel A, et al. A randomised, double-blind, parallel-group study of the safety and efficacy of subcutaneous tocilizumab versus intravenous tocilizumab in combination with traditional disease-modifying antirheumatic drugs in patients with moderate to severe rheumatoid arthritis (SUMMACTA study). Ann Rheum Dis. 2014;73:69-74.

20. Gabay C, Emery P, van Vollenhoven R, et al. Tocilizumab monotherapy versus adalimumab monotherapy for treatment of rheumatoid arthritis (ADACTA): a randomised, double-blind, controlled phase 4 trial. Lancet. 2013;381:1541-50.

21. Maini RN, Taylor PC, Szechinski J, et al. Double-blind randomized controlled clinical trial of the interleukin-6 receptor antagonist, tocilizumab, in European patients with rheumatoid arthritis who had an incomplete response to methotrexate. Arthritis Rheum. 2006;54:2817-29.

22. Nishimoto N, Hashimoto J, Miyasaka N, et al. Study of active controlled monotherapy used for rheumatoid arthritis, an IL-6 inhibitor (SAMURAI): evidence of clinical and radiographic benefit from an X-ray reader-blinded randomised controlled trial of tocilizumab. Ann Rheum Dis. 2007;66:1162-7.

23. Yamanaka H, Tanaka $Y$, Inoue E, et al. Efficacy and tolerability of tocilizumab in rheumatoid arthritis patients seen in daily clinical practice in Japan: results from a retrospective study (REACTION study). Mod Rheumatol. 2011;21:122-33.

24. Burmester GR, Feist E, Kellner H, Braun J, Iking-Konert C, Rubbert-Roth A. Effectiveness and safety of the interleukin 6-receptor antagonist tocilizumab after 4 and 24 weeks in patients with active rheumatoid arthritis: the first phase IIIb real-life study (TAMARA). Ann Rheum Dis. 2011;70:755-9.

25. Yazici Y, Curtis JR, Ince A, et al. Efficacy of tocilizumab in patients with moderate to severe active rheumatoid arthritis and a previous inadequate response to disease-modifying antirheumatic drugs: the ROSE study. Ann Rheum Dis. 2012;71:198-205.

26. Dougados M, Kissel K, Sheeran T, et al. Adding tocilizumab or switching to tocilizumab monotherapy in methotrexate inadequate responders: 24-week symptomatic and structural results of a 2-year randomised controlled strategy trial in rheumatoid arthritis (ACT-RAY). Ann Rheum Dis. 2013;72:43-50.
27. McInnes IB, Thompson L, Giles JT, et al. Effect of interleukin-6 receptor blockade on surrogates of vascular risk in rheumatoid arthritis: MEASURE, a randomised, placebo-controlled study. Ann Rheum Dis. 2015;74:694-702.

28. Burmester GR, Rubbert-Roth A, Cantagrel A, et al. Efficacy and safety of subcutaneous tocilizumab versus intravenous tocilizumab in combination with traditional DMARDs in patients with RA at week 97 (SUMMACTA). Ann Rheum Dis. 2016;75:68-74.

29. Ogata A, Tanimura K, Sugimoto T, et al. Phase III study of the efficacy and safety of subcutaneous versus intravenous tocilizumab monotherapy in patients with rheumatoid arthritis. Arthritis Care Res (Hoboken). 2014;66:344-54.

30. Ogata A, Amano K, Dobashi H, et al. Longterm safety and efficacy of subcutaneous tocilizumab monotherapy: results from the 2-year open-label extension of the MUSASHI study. J Rheumatol. 2015;42:799-809.

31. Schiff $\mathrm{MH}$, Kremer JM, Jahreis A, Vernon E, Isaacs JD, van Vollenhoven RF. Integrated safety in tocilizumab clinical trials. Arthritis Res Ther. 2011;13:R141.

32. Rao VU, Pavlov A, Klearman M, et al. An evaluation of risk factors for major adverse cardiovascular events during tocilizumab therapy. Arthritis Rheumatol. 2015;67:372-80.

33. Grange S, Schmitt C, Ganeshalingam K, Choy EH. Tocilizumab did not significantly increase serum cholesterol levels in healthy subjects. Rheumatology (Oxford). 2014;53(Suppl 1):i95-6.

34. Genovese MC, Smolen JS, Emery $P$, et al. Concomitant use of statins in tocilizumab-treated patients with rheumatoid arthritis with elevated low density-lipoprotein-cholesterol: analysis of five phase 3 clinical trials. Arthritis Rheum. 2008;58(suppl):S785.

35. Arnett FC, Edworthy SM, Bloch DA, et al. The American Rheumatism Association 1987 revised criteria for the classification of rheumatoid arthritis. Arthritis Rheum. 1988;31:315-24.

36. National Cholesterol Education Program (NCEP). Third report of the National Cholesterol Education Program (NCEP) expert panel on detection, evaluation, and treatment of high blood cholesterol in adults (Adult Treatment Panel III) final report. Circulation. 2002;106:3143-421.

37. Arts EE, Popa C, Den Broeder AA, et al. Performance of four current risk algorithms in predicting 
cardiovascular events in patients with early rheumatoid arthritis. Ann Rheum Dis. 2015;74:668-74.

38. Crowson CS, Matteson EL, Roger VL, Therneau TM, Gabriel SE. Usefulness of risk scores to estimate the risk of cardiovascular disease in patients with rheumatoid arthritis. Am J Cardiol. 2012;110:420-4.

39. Barber CE, Smith A, Esdaile JM, et al. Best practices for cardiovascular disease prevention in rheumatoid arthritis: a systematic review of guideline recommendations and quality indicators. Arthritis Care Res (Hoboken). 2015;67:169-79.
40. Peters MJ, Symmons DP, McCarey D, et al. EULAR evidence-based recommendations for cardiovascular risk management in patients with rheumatoid arthritis and other forms of inflammatory arthritis. Ann Rheum Dis. 2010;69:325-31.

41. Solomon DH, Greenberg J, Curtis JR, et al. Derivation and internal validation of an expanded cardiovascular risk prediction score for rheumatoid arthritis: a Consortium of Rheumatology Researchers of North America Registry Study. Arthritis Rheumatol. 2015;67:1995-2003. 